\title{
Art et démocratie
}

Éditorial

Delphine Masset

\section{Introduction}

Depuis les avant-gardes artistiques, dans les milieux initiés, l'art serait perçu, à la suite de ses multiples initiations transgressives, comme étant consubstantiel aux réformes sociales. Cette conception de l'art revêt un caractère profondément idéologique. En effet, comme le retrace brièvement Bell :

«En même temps qu'un engouement pour la nouveauté apparaît l'idéologie, consciemment acceptée, de l'artiste : l'art doit montrer le chemin et servir d'avant-garde. L'idée même d'avant-garde - d'une équipe qui part à l'assaut - indique que l'art et la culture moderne n'admettraient pas d'être le "reflet » d'une structure sociale sousjacente mais qu'ils veulent ouvrir la voie à quelque chose d'absolument nouveau » (BELL : 1979, p.47).

Ainsi, avec la modernité, l'art serait devenu le lieu privilégié pour l'institution d'un nouveau rapport au monde, loin des conventions sociales et des règles de bienséance, en initiant un nouveau rapport aux choses, médié par « l'authenticité » et l'expression individuelle. Et en effet, aujourd'hui, l'art a gagné ses lettres de noblesse: depuis les multiples subversions effectuées par les avant-gardes, l'art est devenu, pour de nombreux analystes, autonome.

Alors que pour l'art moderne l'enjeu principal résidait dans le dépassement des codes de couleurs, des normes d'objectivité de la figuration ou encore du vraisemblable, l'art contemporain a quant à lui motivé la sortie de ses œuvres hors du musée, a été entre autres à l'encontre du bon goût, du droit, de la morale et de l'authenticité (HEINICH 1998). Ainsi, c'est donc à partir du moment où les artistes comprirent l'ampleur du phénomène social qu'était l'art, qu'ils commenceront à s'autonomiser de toute sphère d'influence politique ou sociale (chrétienne, romantique, etc.). Au travers de l'ensemble de ces actes transgres- 
sifs, l'art commettra, en termes psychanalytiques, le «meurtre du père »" C'est alors et alors seulement qu'il deviendra autonome et qu'il n'aura plus de compte à rendre à personne.

Pour ces raisons, aujourd'hui, l'art représente de manière emblématique le cénacle où chacun pourrait prendre librement la parole. Libéré de toutes les contraintes et formes d'autorité extérieure, lieu par excellence de la valorisation de l'originalité voire de la marginalité, l'art nous emmènerait loin de l'espace minimal de parole qu'est le " dèmos » ${ }^{2}$ aujourd'hui, loin des standards d'expressivité (le vote) que nous aurions pour nous faire entendre, loin, encore, de ce lieu où le numéraire prend le pas sur l'inquantifiable du numéro, où la souffrance ne peut être comptabilisée. Ainsi, là où se joue l'humain, dans notre dèmos, certains diront que rien ne peut en être dit.

\section{Menaces et suggestions}

Ainsi, l'art, en ce qu'il valorise le singulier et sacralise la différence, tendrait à vouloir donner la parole à tout le monde et de manière générale, à rendre compte de voies-voix alternatives. Loin du poids contraignant de la société, nous aurions ainsi affaire à un topos ouvert à l'autre, à l'altérité.

Cependant, la sacralisation de l'expression individuelle est mise en concurrence par le pouvoir de tout un chacun à prendre la parole. Dans ce jeu de balancier, le dèmos n'est pas toujours gagnant. L'ouverture à l'autre et l'écoute attentive aux voies-voix alternatives se soldent par une incapacité à «mettre ensemble ». L'accumulation des points de vue individuels ne font pas société : comme Durkheim nous le disait «le tout est bien plus que la somme des parties ».

L'artiste qui sacralise l'expression individuelle et symbolise le mythe démocratique n'est-il pas justement dans cette optique le contrepoids d'une société où se multiplient les ego, sans que jamais ne soient mis en résonnance les différents points de vue? Comme convergence à ce système schizophrénique (où le mythe occulte la réalité des choses) on découvre la multiplication des petitsfascismes, modes de pensée qui se donne à voir dans le pouvoir de décider pour les autres ce qu'est la "vérité», en sacralisant leur expression individuelle. C'est pourquoi il nous faut faire preuve d'une vigilance accrue vis-à-vis de l'art

\footnotetext{
${ }^{1}$ Le « père » étant entendu comme cet objet d'art dont l'aura est indubitable et dont la valeur est essentialisée.

${ }^{2}$ Par « dèmos », nous entendons la totalité des membres de la Cité.
} 
contemporain et des autres mythes fondateurs d'un dèmos émancipé : il n'est pas d'art ou de modèle de participation politique qui soient affranchis du risque de servir des intérêts particuliers. Combien de performances collectives artistiques ou combien de tables-rondes n'ont-elles pas flouées les acteurs sur la réelle prise en compte de leur voix - le réel poids de leur intervention?

Par ailleurs, en lien avec la progressive conquête de l'autonomie de l'art vis-àvis des autres sphères d'influence, il nous semble justifié de nous demander si c'est à ce dèmos que nous aspirons. En effet, les artistes peuvent aujourd'hui tout raconter sans que personne n'ait rien à dire : «ni élection, ni jugement, ni concurrence, ils n'ont à répondre de rien, c'est même ce qui les définit parfaitement. En ce sens, ils ont acquis l'autonomie absolue dont les artistes modernes rêvaient » (HENNION 2010, p.4). A contrario d'un tel système complètement libéralisé, nous pensons que le dèmos doit s'accompagner d'instances délibératives afin que le collectif bénéficie d'une capacité de juger. Certes, la tâche est difficile en art, mais l'écoute du public - ou de son silence - pourrait, pourquoi pas, être un bon indicateur.

Outre cela, le sentiment répandu dans la société que le dèmos ne s'atteindrait que par une autonomisation totale des individus nous semble n'être à ce titre que le reflet d'une conception depuis longtemps encrée dans le monde de l'art. On peut cependant douter d'une possibilité totale d'autonomisation quand on entrevoit à peine les liens tissés entre les hommes (invisibles la plupart du temps, certes) au travers de nos rapports marchands. Reconnaitre nos interdépendances devrait être en ce sens érigé au rang des vertus démocratiques. Ainsi, il serait judicieux de se demander si la figure de l'art et de l'artiste autonome n'entretiendrait pas le mythe de l'individu contemporain qui s'auto-engendre. La figure de l'artiste ne serait alors que l'autre face de l'invention méritocratique (basée sur la conviction que les conditions d'existence seraient également distribuées à la naissance).

Malgré le cynisme apparent de ces mises en garde, il nous faut cependant avouer que tous ces mythes ne sont pas détournés et que parmi eux, des expériences tout à fait concluantes sont possibles. Sans pousser au conservatisme, notre message est donc celui-ci : il nous faut être attentif aux tyrannies de la participation et de l'expression individuelle; de nombreuses fois, celles-ci ne servent que des intérêts contraires, à savoir, l'établissement d'une voie-voix audessus des autres, le dogmatisme des intérêts particuliers sur l'intérêt général. Pour ces raisons, il faut être vigilant vis-à-vis des relations entretenues entre l'art et la démocratie. 


\section{L'art et le politique}

Pour ceux qui en doutaient encore, la trac e du politique dans l'art est donc omniprésente. Indubitablement, l'art est politique : ne serait-ce que parce qu'il propose un autre régime d'expression que les modes habituels d'expression politique, il dit quelque chose sur le politique.

Si nous avons l'habitude de définir le politique au travers d'un « régime de discours politique » qui nécessite prise de parole, joute verbale, argumentation et débat, il est envisageable de situer celui-ci bien au-delà de ce régime et de l'enraciner dans différentes formes d'expressions artistiques. L'enceinte politique, bien plus que d'être circonscrite dans les institutions de pouvoir, peut s'étendre à d'autre mode d'expressivité que l'expressivité politique (avec ses grilles de lecture, sa conception du bien commun, etc.), comme celles propres à la sensibilité artistique. Par exemple, si les romantiques disaient au travers de leur art l'importance qu'ils donnaient à la nature et au mode de vie " bohème », ils racontaient, de facto, l'indifférence qu'ils pouvaient accorder au régime d'accumulation ou à la volonté de maitrise de la société occidentale. Outre le régime d'expression, l'art est politique pour toutes les valeurs qu'il véhicule en son sein : conception de l'art et considération sur l'artiste, comme nous l'avons suggéré en amont, ne sont pas en reste dans l'appréhension politique que nous devons avoir de l'art.

Ces quelques suggestions ne sont qu'une ouverture, de multiples autres éléments devraient être ajoutés à la liste des traversées du politique dans l'art. C'est ce que nous découvrirons tout au long de ce numéro consacré au lien entre l'art et la démocratie. Et c'est parce que l'art est politique que la question démocratique peut (doit ?) être posée, et cela, à tous les niveaux où se joue l'art.

Mais pourquoi ce questionnement en particulier? Pourquoi ce lien incongru pour certains ? Voici la réponse, probablement partiale et militante : parce que l'art est le lieu par excellence où semblent s'exercer les entreprises les plus fécondes, mais aussi les dérives les plus patentes des diverses tentatives démocratiques. Lieu vers lequel se tournent tous les espoirs, enceinte mythique gorgée d'idéologies multiples, l'art favorise l'expérimentation, autant qu'il est un lieu propice pour les manipulations politiques en tout genre. C'est pour cette double raison que l'art nécessite toute notre attention.

\section{L'art et son insertion dans le système social et économique}

La question à l'origine de ce numéro d'Émulations était celle-ci : «que peut-on dire aujourd'hui sur les liens entretenus entre l'art et la démocratie? L'art et ses pratiques consœurs sert-il le dèmos, le peuple ; libère-t-il, transcende-t-il les hié- 
rarchies? Ou est-il au service d'autres causes? Quels en sont les intentions et les pièges ? Ou encore, à quoi d'autre pourrait-on aspirer? »

En effet, l'art, par le biais de la peinture, du graphisme, des performances, de l'architecture ou encore du design semble aujourd'hui aussi bien servir l'industrie culturelle et les entreprises marchandes qu'évoquer le Beau et le Bien, accompagner la création de sens et l'invention de fables, proposer des utopies ou encore permettre la critique sociale. À une époque où les frontières entre objet d'art et objet commercial, expression et image, sont de plus en plus brouillées, la question du statut de l'art dans nos sociétés nous semblait ainsi se poser plus que jamais.

Les prétentions critiques de l'art peuvent parfois n'être que de purs idéaux et entretenir éventuellement une forme d'idéologie. La sociopolitique marxiste a depuis bien longtemps mis en exergue le fait que l'art et la culture n'était que le reflet d'une idéologie, qu'un moyen d'exercer une domination au travers d'une structure de sens. On serait ainsi tenté de dire que l'art peut jouer un rôle de confirmation esthétique de l'ordre social. En effet, l'art n'est pas forcément un outil d'émancipation sociale et démystifier son rôle parait être un passage obligé. Certains auteurs ${ }^{3}$ ont prétendu l'existence d'une autonomie grandissante de la sphère artistique ; il semble aujourd'hui nécessaire de s'en assurer. Si certes, les valeurs soutenues au sein du monde artistique semblent être celles de l'indépendance, du désintérêt et de l'individualité, on peut à juste titre se demander, par exemple, "qui fait l'art». En effet, les conditions matérielles de productions de l'art sont intrinsèquement sous-tendues par la présence, sur le marché de l'art, de galeristes, de collectionneurs ou encore du pouvoir public. Il n'y a pas d'art sans public comme il n'y a pas d'objet d'art sans acquisiteur. L'art, en sa qualité d'objet social qui se définit performativement, n'est donc pas autonome. Il est aussi dépendant de ses potentiels acquisiteurs que sa valeur est détenue par l'ensemble de ses critiques d'art. Ce sont eux qui font l'art et qui contribuent à son système d'échange.

Ainsi, si l'art semble, de prime abord, délié de tout type de normativités ou de phénomènes sociaux, de nombreux éléments concordent à nous faire penser que cela n'est, au fond, pas le cas. La présence des industries culturelles, des médias ou des pouvoirs politiques dans la diffusion ou la réception des œuvres d'art nous pousse à être dubitatifs quant à une possible autonomie de la sphère artistique par rapport au monde social.

\footnotetext{
${ }^{3}$ Bourdieu en fait partie. Weber l'avait prédit. Habermas en avait parlé.
} 
Par ailleurs, à l'opposé des considérations pessimistes qui viennent d'être faites, en ce que l'art est un laboratoire incessant, il débouche régulièrement sur des expériences qui transcendent les cadres établis du dèmos et qui viennent largement enrichir les pratiques, en ouvrant sur de nouvelles perspectives démocratiques.

Pour analyser les divers liens existants entre art et démocratie, nous nous pencherons sur les trois niveaux d'élaboration présents dans l'art que sont sa confection, sa diffusion et sa réception. La présente livraison d'Émulations sera donc scindée en trois volets : un premier sur le contenu politique de l'art; un second sur l'intervention publique ou privée dans la diffusion de l'art, et un troisième sur la réception de l'art et ses conséquences politiques.

\subsection{Premier volet : Le contenu politique de l'art}

Au travers de notre numéro, nous débattrons de deux types de propositions artistiques relativement distinctes : un premier type, caractéristique d'un "contexte de transition et de revendication démocratique» (ATERIANUS, dans ce numéro), et un second type, propre aux problématiques occidentales de nos sociétés démocratiques libérales.

Le premier type a donc trait aux pays non occidentaux. Liliane Anjo ${ }^{4}$ nous explique ainsi, qu'en Iran, le pouvoir politique réduit encore tellement les libertés que les tensions en œuvre dans l'art sont toute autre que celles produites par l'autonomie artistique : il s'agit davantage de signaler, entre les lignes des déclamations successives, quelle autre vie que celle administrée par le gouvernement, serait désirable ou encore de quels maux souffre la société iranienne. Le simple fait de pouvoir s'exprimer ou de pouvoir décrire l'existant suffit à donner du sens à la pratique artistique iranienne. C'est avec le même objectif que les rappeurs gabonais, décrits par Alice Aterianus ${ }^{5}$, « orchestrent et bâtissent parallèlement par leurs performances musicales des espaces d'expression démocratiques libérés des contraintes et des cadres verrouillant les discours dans l'espace public ». Ainsi, nous avons bien affaire à un art libérateur qui participe aux avancées démocratiques.

Dans ce numéro, le deuxième type de propositions, qui prend pied dans un environnement occidental, seront emblématiquement représentées par l'art par-

\footnotetext{
${ }^{4}$ Lilian Anjo : « De la brèche créatrice à l'imaginaire libéré : L'art dramatique en Iran ».

${ }^{5}$ Alice Aterianus : "Quand l'indigénisation du rap réinvente les langages du politique. Lespratiques musicales urbaines : des nouvelles voix(es) démocratiques au Gabon?
} 
ticipatif et relationnel (auquel Catherine Duschesneau ${ }^{6}$, Alice Laguarda ${ }^{7}$ ainsi que de Johanna Renard ${ }^{8}$ nous introduirons). L' «art relationnel» vu comme «ensemble de pratiques artistiques qui prennent comme point de départ l'ensemble des relations humaines et leur contexte social» (BOURRIAUD : 1998, p.117) proposera en effet une alternative au monde déshumanisé de l'art contemporain ou de la sphère marchande. Son «esthétique se propose de la sorte comme une manière de formation alternative à la vie postmoderne : il s'agit d'«apprendre à mieux habiter le monde » en investissant la sphère relationnelledans une économie non standard du quotidien vécu » (Alliez : 2008, pp.121-125.). Proposition artistique à la vie postmoderne, l'art relationnel sera donc une nouvelle forme de réinvention du quotidien.

La place laissée à l'utopie démocratique et participative est donc centrale : c'est elle qui permettra, par le truchement d'une intégration de tous et de toutes, et par l'élaboration de nouvelles pratiques plus libérées, de redonner du sens au monde. Et dans ce cadre, le contexte mondialisé et marchand semblera être un point d'accroche pour tous : c'est à partir de lui que rebondissent beaucoup d'artistes, contre lui que de nombreuses propositions artistiques sont faites.

Élodie Lapp nous relèvera à ce sujet du fait que c'est «à l'heure de la mondialisation et de la culture en réseau, [que] de plus en plus de pratiques artistiques se dirigent vers une conception participative, interactive, voire collaborative». L'auteure nous apprendra donc que derrière ces œuvres assez atypiques, c'est bien la poursuite de l'idéal démocratique qui se donne à voir: "qu'il s'agisse d'inclure le public dans le processus même de production ou de faire émerger des formes le sollicitant en tant qu'activateur de sens, il apparait bel et bien que la tendance soit de donner, si ce n'est la parole, tout au moins une légitimité de présence à tout monde ».

De manière similaire, l'expérience du Grand Union's Improvisation Laboratory dont nous fait part Johanna Renard dans sa contribution, est emblématique d'un tel idéal démocratique. Ici, l'improvisation sera considérée «à la fois comme une forme artistique et une forme de vie, afin de transcender les hiérarchies et élaborer un véritable fonctionnement communautaire démocratique ».

Il faut donc comprendre qu'à l'image d'une colonisation de l'espace quotidien par l'économique ou encore, à l'image d'une banalisation (voir d'une occulta-

${ }^{6}$ Catherine Duchesneau : « De la participation en art. L’Écomusée du fier monde comme alliance entre l'art et la participation citoyenne».

${ }^{7}$ Alice Laguarda : «Art et politique, reconquérir usage et expérience ».

${ }^{8}$ Johanna Renard : "The Grand Union's Improvisation Laboratory. La démocratie en mouvement ». 
tion) de nos actes politiques (entre autres, de consommation), l'art relationnel tentera de remettre du sens là où il s'est évaporé : entre les gens eux-mêmes. Les hommes étant devenus les terminaux des systèmes technoscientifiques (ARIÈS : 2010, p.125) qui fonctionnent par de successives sous-traitances (au travers du cycle de production-consommation, qui fait transiter un même produit par maints pays et intermédiaires), il nous est aujourd'hui bien difficile de (re)connaitre notre interdépendance aux autres. Si l'art participatif et relationnel est entre autres une réponse à la crise du lien social, il fustige aussi indirectement la conversion du politique par l'économique et donc, l'hypertrophie du lien social qui en résulte. En ce sens, l'art participatif et relationnel sera marqué par les problématiques de son système politique et de son époque.

Il nous faut alors nous demander si lorsque pour l'art participatif « il ne s'agit plus aujourd'hui de défendre de grandes utopies, mais bien de prendre position à des niveaux plus infimes, plus locaux, en somme à des niveaux micropolitiques et d'entrer en rapport avec autrui » (DUSCHESNEAU, dans ce numéro), il ne faut pas sonner la fin du «dèmos ». En effet, les micro-utopies sont autant de manières de faire croire que nous ne serions pas face, simplement, à l'emprise de quelques-uns sur tous. En ce sens, l'art relationnel maintient le déficit démocratique actuel par l'entretien de l'illusion de l'efficacité d'une reconstruction du dèmos à petite échelle, et de manière expérimentale. Par ailleurs, il faut craindre que cet idéal ne puisse être atteint à partir du moment où «le mécénat privé tend aujourd'hui à se généraliser, voire à opérer comme un effet de mode et ce, bien sûr, sous couvert de démocratisation » (LAPP, dans ce numéro).

En effet, nous verrons aussi, dans ce numéro d'Émulations, en quoi l'art relationnel peut être sujet à diverses instrumentalisations, quand il n'est tout simplement pas une mascarade. Pensons par exemple à l'exposition de la galerie Z33 à laquelle nous avons assisté et qui présenta une œuvre relationnelle ${ }^{9}$ où des livres intitulés Stealthis book étaient mis à disposition... mais ne pouvaient pas être volés. L'invitation libertaire à l'appropriation de l'objet et l'appel à l'interaction avec le public n'étaient en fait qu'une pure mise en scène, que le simple simulacre d'un rapport plus «démocratique » entre l'artiste et son public ${ }^{10}$.

${ }^{9}$ Galerie Z33, Exposition « Steal this book » de Dora García, mars 2010.

${ }^{10}$ En ce sens, il sera utile de déplacer notre regard du contenu démocratique de l'œuvre d'art aux lieux de sa diffusion et des politiques qui le motivent. Toute une série d'articles, présenté dans le point suivant, nous le permettrons. 
Par ailleurs, il faut reconnaitre qu'il appartient aussi à l'art de montrer la beauté du geste, la fragilité et l'imperfection des tentatives en prenant le risque de se « tromper » ou d'être maladroit, comme nous le suggère Johanna Renard. Dans ce sens, le «dèmos » renvoie davantage à l'abolition des hiérarchies entre les arts et dans l'art. Peu importe alors la portée politique de l'art et seule importe sa portée esthétique...

A terme, et au fil des divers articles présents dans le présent numéro d'Émulations, ce sera au lecteur de se faire son propre point de vue. Nous pourrons simplement arguer d'avoir proposé un rapide coup d'œil sur le paysage artistique des mondes occidentaux d'aujourd'hui.

\subsection{Deuxième volet : L'intervention publique ou privée dans la diffusion de l'art}

Comme nous venons de le suggérer, outre le contenu démocratique de l'art, un autre approche peut être envisagée pour aborder le lien entre art et démocratie : celle qui lie les politiques publiques ou les entreprises privées à l'art. C'est ainsi qu'Élodie Lapp $^{11}$ et Flavie Holzinger ${ }^{12}$ nous parlent des politiques de promotion publiques de l'artiste ou encore de l'usage de l'art dans le Monde diplomatique.

C'est à l'aune d'une telle réflexion que nous pourrons constater à quel point les politiques publiques et les entreprises privées oscillent entre promotion de l'art et instrumentalisation de celui-ci ou encore entre démocratisation culturelle et marchandisation artistique. Comme nous le rappellera Élodie Lapp, il n'y a par exemple qu'un pas entre les politiques de promotion artistique et les politiques d'attractivité des villes.

D’un autre côté, comme nous l'explique Flavie Holzinger, le cas de l'utilisation de l'art dans le Mondediplomatique "permet une prise de conscience du pouvoir politique auquel peut prétendre l'œuvre d'art même si son producteur ne l'a pas conçue dans ce sens ». Ainsi, entre une instrumentalisation de l'art dans un but purement économique (comme dans le cas de l'attractivité culturelle des villes) et une instrumentalisation du pouvoir politique de l'œuvre dans un but journalistique et démocratique, il y a une différence à faire. En effet, « le Monde diplomatique participe au décloisonnement de l'art. Il a initié une

\footnotetext{
${ }^{11}$ Élodie Lapp : «L'art contemporain et la démocratie : Des œuvres interactives aux politiques de promotion publique de l'artiste».

${ }^{12}$ Flavie Holzinger : «Art et démocratie : L'usage de l'art dans Le Monde diplomatique ou la pratique picturale d'un journal engagé ».
} 
nouvelle conception du rapport entre l'art et le public. Il utilise l'art comme un moyen, un outil de lutte qui dénonce ou sert une cause».

\subsection{Troisième volet: La réception de l'art et ses conséquences politiques}

Au moment de la production (du contenu) de l'art et de sa diffusion (de sa promotion publique, par exemple), succède un troisième moment, celui de la réception. Ainsi, dans l'exercice d'une détermination du lien entre art et démocratie, un troisième point de vue aurait pu être développé. Il aurait fallu alors s'interroger sur la place laissée au spectateur, une place qui oscille entre passivité du réceptacle et activité du récepteur.

En aval de la chaine de production et de diffusion, on peut donc s'interroger sur la place de l'imaginaire laissée dans l'image. A-t-on affaire à une image-idole qui laisse sans voix et qui ne suscite pas la parole ou a-t-on affaire à une image discutée, qui suggère le sens plutôt que l'impose ou le sature, qui éveille le sentiment plutôt que l'annihile ? Ainsi, selon le cadrage préexistant à une interaction avec une œuvre d'art (surinformation médiatique ou pas), on promouvra un art dont la réception sera pleinement passive, à l'image d'un enseignement maittre-élève, ou un art qui donne à parler et à réfléchir. En ce sens, les entreprises médiatiques qui standardisent la réception d'une œuvre et homogénéisent le rapport entretenu avec celle-ci, font courir à leur perte le potentiel démocratique d'une œuvre d'art. Tous se taisent quand les médias parlent: chacun n'aurait donc pas la légitimité de la parole « juste» face à une œuvre d'art. La crainte de Marie-José Mondzain est en ce sens que « la standardisation de la vision [qui] vient lentement anéantir l'énergie créatrice du regard, les modèles dominants imposent un imaginaire qui indexe la reconnaissance intersubjective sur le partage d'objets identiques relevant de la consommation iconique» (MONDZAIN : 2007, p.112). Ainsi, l'auteure replace le débat autour de la problématique de la consommation. Parce que consommer ne permet pas au spectateur d'apprendre à se connaître « au travers de », de se découvrir " par», il faut chercher aujourd'hui à faire de l'œuvre le lieu de la relation afin de faire advenir l'ouverture et l'épanouissement de l'être. Voir revient alors à faire naitre le désir (de sentir, de découvrir, d'avoir des émotions) plutôt que de fermer le sens (de savoir). Le regard, le mouvement des yeux, l'acte de vision doit ouvrir davantage plutôt que de combler. L'art « digne » ou « démocratique » devient alors celui qui redonne sa juste place à l'individu, celui qui lui permet de faire épanouir sa propre sensibilité. Ainsi, rendre possible le dèmos, c'est aussi donner la parole au spectateur, lui rendre son pouvoir de voir, de sentir, de toucher. 


\section{Conclusions}

Au long de ce numéro d'Emulations, le lecteur pourra donc évaluer le lien entretenu entre l'art et la démocratie au travers de sept contributions, que le hasard veut toutes féminines. Ces contributions balisent l'appréhension possible de ce lien à la fois au niveau du contenu de l'art « démocratique » et des procédés dits démocratiques de diffusion de l'art.

Nous avons ainsi peu abordé les questions plus philosophiques de la place de la parole du spectateur dans l'art ou encore des coordonnées des politiques publiques nationales ou internationales en matière artistique (telles que le choix des critères déterminants pour l'allocation de subsides). Nous n'avons en ce sens qu'effleuré la problématique tout en permettant au lecteur de se faire une idée de celle-ci au niveau européen et extra-européen.

Nous pourrons cependant retenir à ce niveau le caractère profondément ambivalent de l'art. Celui-ci peut en effet aussi bien servir les entreprises démocratiques que les desservir (souvent, plus que ce qui n'y parait). L'art, en effet, peut être instrumentalisé à des fins communicationnelles ou promotionnelles, il peut servir le spectaculaire, le souci d'attractivité.

Ainsi, outre le contenu de l'art, tout dépend de ce qu'il en sera fait - à la fois par les artistes et par les autorités en place. Tout dépendra, dans ce sens, du système dans lequel il prendra forme : comme nous l'avons vu, le rôle de l'art au Gabon, en Iran ou en Europe et au Canada ne sera pas similaire ; les menaces du promotionnel n'étant pas comparables.

Il s'agira donc avant tout, comme le plus souvent lorsque nous prenons conscience d'un risque auquel mène une œuvre d'art ou un genre artistique, de consacrer toute son attention à éviter les dérives liées aux usages qui lui sont propres. Ceux-ci resteront toujours aussi nombreux que les œuvres d'art ellesmêmes.

\section{Références bibliographiques}

Alliez E. (1998), «Post-scriptum sur l'esthétique relationnelle: Capitalisme, schizophrénie et consensus ». InMultitudes, vol.3 n³4, pp.121-125.

Ariès P. (2010), La simplicité volontaire contre le mythe de l'abondance. Villeurbanne: Golias.

Bourriaud N. (1998):Esthétique relationnelle. Paris: Presse du réel.

Heinich N. (1998), Le triple jeu de l'art contemporain. Paris: Éditions de Minuit.

Mondzain M. (2007), Homo spectator. Paris: Bayard. 
Hennion A. (2010), «L'art contemporain est-il politique? Création, marché, public ». In Collectif (2010), Art, éducation, politique. Actes du colloque international d'Angoulême, 2224 octobre 2009. Paris: Sandre. 